\title{
A new application for Coomassie Brilliant Blue agar: detection of Aeromonas salmonicida in clinical samples
}

\author{
Nancy M. Markwardt, Yvonne M. Gocha, George W. Klontz
}

Department of Fish and Wildlife Resources, College of Forestry, Wildlife and Range Sciences, University of Idaho, Moscow, Idaho 83843, USA

\begin{abstract}
Coomassie Brilliant Blue (CBB) agar was examined to assess its applicability as a differential medium for Aeromonas salmonicida. The characteristics of 6 species of bacteria, including fish pathogens, on the CBB medium, in mixed culture, and upon isolation from asymptomatic carrier fish, were determined. Results indicate that this medium can be used to differentiate $A$. salmonicida in mixed bacterial populations from clinical samples.
\end{abstract}

\section{INTRODUCTION}

Aeromonas salmonicida, the etiological agent of furunculosis, infects a broad range of salmonid species and is responsible for substantial grow-out and postrelease mortality in anadromous and resident stocks (Klontz 1968, Scallan \& Smith 1985). Presumptive identification of $A$. salmonicida is based primarily on its ability to produce a soluble brown pigment when grown on tryptic soy agar. However, the use of this chromogenic characteristic for differential identification has been shown to be unreliable. Pigment production by $A$. salmonicida in mixed culture is apparently inhibited by the other bacteria (Austin \& Austin 1987). In addition, several achromogenic strains of $A$. salmonicida (Smith 1963, Paterson et al. 1980) and a chromogenic strain of Aeromonas hydrophila (Ross 1962) have been isolated.

Coomassie Brilliant Blue (CBB) agar was developed by Udey (1982). This medium was used in modified form by Wilson \& Horne (1986) for the differentiation of $A+$ and A- strains of Aeromonas salmonicida and has been successfully used for this purpose (Cipriano \& Bertolini 1988). The goal of this study was to determine if the Udey form of this medium could be used as a differential medium for clinical identification of A. salmonicida.

\section{METHODS}

Coomassie Brilliant Blue agar preparation. $100 \mathrm{mg}$ $\mathrm{l}^{-1}$ Coomassie Brilliant Blue dye (C.I. 42655) was added to $44.0 \mathrm{~g}$ tryptic soy agar. After autoclaving $15 \mathrm{~min}$ at $1.05 \mathrm{~kg} \mathrm{~cm}^{-2}$, ca $18 \mathrm{ml}$ were poured into $100 \times 15 \mathrm{~mm}$ disposable culture dishes.

Pure culture analysis. Twenty-three bacterial isolates were examined for colony color on $3 \mathrm{CBB}$ medium replicates following $48 \mathrm{~h}$ incubation at 17 and $21^{\circ} \mathrm{C}$ (Table 1).

Mixed culture analysis. Individual tubes of tryptic soy broth were inoculated with selected bacterial colonies and incubated at $21^{\circ} \mathrm{C}$ for $36 \mathrm{~h}$. The Aeromonas salmonicida isolates selected for analysis were FHM 031 and FHM 034, along with representative isolates of other bacterial cultures (A. hydrophila FHM 004, 014, 033, 036; Yersinia ruckeri-FHM 027; Vibrio anguillarum - FHM 009; Enterobacter aerogenes - BAC 01; and Escherichia coli - BAC 02). Two different broth cultures were mixed, streaked for isolation on $\mathrm{CBB}$, and the individual colony types were streaked on new CBB plates. Selected colonies, identified by color as being $A$. salmonicida, were confirmed using direct immunofluorescence (FAT) (Klontz \& Anderson 1968).

Tests with clinical samples. Asymptomatic carriers of Aeromonas salmonicida (FHM 034) were established via gastric intubation of juvenile spring chinook salmon (Markwardt \& Klontz in press). The large intestine was removed from 50 randomly selected fish 4 d post-infection. Intestines from individual fish were weighed and homogenized in $0.5 \mathrm{ml}$ sterile water with a $10 \mathrm{ml}$ Wheaton motor-driven tissue homogenizer. The homogenized material was then diluted 10 -fold, 4 times, in $0.85 \%$ sterile saline. Three drops $(0.10 \mu \mathrm{l})$ of 
Table 1 Bacterial isolates used for evaluating Coomassie Brilliant Blue medium. Sources: AL: Abbott Laboratories, Chicago, IL, USA; ATCC: American Type Culture Collection, Rockville, MD, USA; BC: Pacific Biological Station, Nanaimo, B.C., Canada; CST. Clear Springs Trout Co, Buhl, ID, USA FHM: Fish Health Management Laboratory, Moscow, ID USA; UI: University of Idaho, Dept. of Bacteriology, Moscow, ID. USA; WDF: Washington Department of Fisheries, Olympia, WA, USA

\begin{tabular}{|lll|}
\hline Bacterial isolate & Isolate no. & Source \\
\hline Aeromonas salmonicida & FHM 008 & BC \\
& FHM 026 & ATCC 33659 \\
& FHM 031 & CST \\
& FHM 034 & CST \\
& FHM 037 & WDF \\
& FHiv 038 & WDF \\
& FHM 039 & WDF \\
& FHM 040 & WDF \\
& FHM 041 & WDF \\
Feromonas hydrophila & FHM 042 & WDF \\
& FHM 004 & FHM \\
& FHM 014 & AL \\
& FHM 024 & ATCC 14715 \\
& FHM 033 & CST \\
Yersinia ruckeri & FHM 036 & CST \\
& FHM 007 & BC \\
& FHM 020 & CST \\
Vibrio anguillarum & FHM 021 & CST \\
Vibrio ordalii & FHM 027 & ATCC 29473 \\
Enterobacter aerogenes & FHM 009 & BC \\
Escherichia coli & FHM 010 & BC \\
& BAC 01 & UI \\
& BAC 02 & UI \\
\hline & & \\
& &
\end{tabular}

each dilution were placed in 3 locations on plates of CBB medium. The plates were then incubated for $48 \mathrm{~h}$ at $21^{\circ} \mathrm{C}$. Representative dark blue colonies were randomly selected for confirmation of identity by FAT.

\section{RESULTS}

Pure culture analysis. All of the Aeromonas salmonicida colonies were dark blue with the exception of achromogenic strain FHM 026. This isolate was originally medium blue; however, after several serial passes through chinook salmon, the colony color exhibited on CBB was dark blue (Table 2).

Mixed culture analysis. Isolation of Aeromonas salmonicida based on colony color was successful with both isolates (FHM 031 and FHM 034). The dark blue colonies from the following combinations were positive for A. salmonicida using FAT:FHM 031 with Yersinia ruckeri or with A. hydrophila (2 strains); FHM 034 with $Y$. ruckeri or with $A$. hydrophila (3 strains).

Tests with clinical samples from asymptomatic carriers. Two colony types were isolated from the asymp-
Table 2. Pure isolate colony color results when grown on the Coomassie Brilliant Blue medium

\begin{tabular}{|lc|}
\hline Bacterial species & Colony color \\
\hline Aeromonas salmonicida & Dark blue \\
Aeromonas hydrophila & Medium blue \\
Yersinia ruckeri & Light blue \\
& (FHM 027-medium blue) \\
Vibrio anguillarum & Blue green \\
Vibrio ordalii & Light blue \\
Enterobacter aerogenes & Light blue \\
Escherichia coli & Light blue \\
\hline
\end{tabular}

tomatic carrier fish. The dark blue colonies, suspected to be Aeromonas salmonicida, were positively identified as such using FAT. The other colony type, medium blue, was identified as $A$. hydrophila using API 20 E bacteriological identification strips.

\section{DISCUSSION}

The Aeromonas salmonicida isolates were the only cultures appearing dark blue when grown on CBB. The ability of this medium to differentiate $A$. salmonicida from other bacterial microflora present in fish is a valuable application of this medium. Rarely do samples from diseased or asymptomatic fish contain a single bacterial species. This ability to quickly identify $A$. salmonicida in mixed culture is even more powerful when one considers the ease of differentiation from the chromogenic strain of $A$. hydrophila.

The CBB medium is only effective for Aeromonas salmonicida differentiation when the isolate is $\mathrm{A}+$ (Wilson \& Horne 1986). The presence of the $A+$ layer protein is associated with the ability to autoagglutinate, to adhere to host tissue, and the ability to establish infection (Udey \& Fryer 1978, Trust et al. 1983). Thus, the majority of the clinical isolates of $A$. salmonicida are A +. Although there are virulent A- strains (Johnson et al. 1985), these are the exception rather than the rule.

This medium is differential and presumptive. Thus, identification based on the colony color reaction on the CBB medium should be followed with definitive identification techniques, such as those based on serological and biochemical characteristics. This medium can be used in diagnostic and epizootiological work to presumptively identify Aeromonas salmonicida in samples from clinically ill or asymptomatic fish and from water samples; it can also be used to determine the prevalence of the pathogen in such samples.

Acknowledgements. The authors thank the Idaho State Board of Education for their financial support of this research project. We also thank Dr Jim Congleton and Dr George Teresa for 
reviewing the manuscript. This is Idaho Forest, Wildlife, and Range Experimental Station Contribution No. 371.

\section{LITERATURE CITED}

Austin, B., Austin, D. A. (1987). Bacterial fish pathogens: disease in farmed and wild fish. Ellis Horwood, Chichester, England

Cipriano, R. C., Bertolini, J. (1988). Selection for virulence in the fish pathogen Aeromonas salmonicida, using Coomassie brilliant blue agar. J. Wildl. Dis. 24: 672-678

Johnson, D. M., Tatner, M. F., Horne, M. T (1985). Autoaggregation and extracellular A-layer protein in Aeromonas salmonicida. Aquaculture 46: 163-166

Klontz, G. W. (1968). Oral immunization of coho salmon against furunculosis. In: Progress in sport fishery research. Resour. Publ. Bur. Sport Fish Wild. U.S. 39: 81-82

Klontz, G. W., Anderson, D. P. (1968). Fluorescent antibody studies of Aeromonas salmonicida. Bull. off. Int. Epiz. 69: 1149-1157

Markwardt, N. M., Klontz. G. W. (in press). Evaluation of four methods to establish asymptomatic carriers of Aeromonas salmonicida in juvenile spring chinook salmon. J. Fish Dis.

Paterson, W. D., Douey, D., Desautels, D. (1980). Relationships between selected strains of typical and atypical Aeromonas salmonicida, Aeromonas hydrophila, and Haemophilus piscium. Can. J. Microbiol. 26: 588-598

Ross, A. J. (1962). Isolation of a pigment-producing strain of Aeromonas liquefaciens from silver salmon (Oncorhynchus kisutch). J. Bacteriol. 84: 590-591

Scallan, A., Smith, P. R. (1985). Control of asymptomatic carriage of Aeromonas salmonicida in Atlantic salmon smolts with flumequine. In: Ellis, A. E. (ed.) Fish and shellfish pathology. Academic Press, New York, p. 119-127

Smith, I. W. (1963). The classification of 'Bacterium salmonicida'. J. gen. Microbiol. 33: 263-274

Trust, T. J., Kay, W. W., Ishiguro, E. E. (1983). Cell surface hydrophobicity and macrophage association of Aeromonas salmonicida. Curr. Microbiol. 9: 315-318

Udey, L. R. (1982). A differential medium for distinguishing Alr ${ }^{+}$from $\mathrm{Alr}^{-}$phenotypes in Aeromonas salmonicida. In: Proceedings of the 13th Annual Conference and Workshop and 7th Eastern Fish Health Workshop. Intl Assoc. for Aquat. Animal Med., Baltimore, Maryland, p. 41

Udey, L. R., Fryer, J. L. (1978). Immunization of fish with bacterins of Aeromonas salmonicida. Mar. Fish. Rev, 40: $12-17$

Wilson, A., Horne, M. T (1986). Detection of A-protein in Aeromonas salmonicida and some effects of temperature on A-layer assembly. Aquadulture 56: 23-27 